\title{
Nrf2 regulates collective cancer migration by modulating the hybrid epithelial/mesenchymal phenotype
}

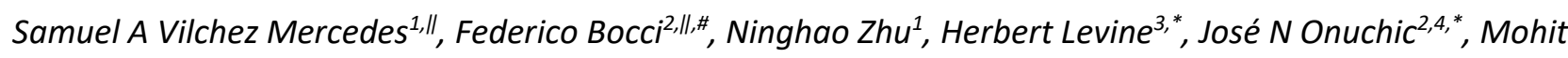
Kumar Jolly, ${ }^{5,}$, and Pak Kin Wong ${ }^{1,6, *}$

${ }^{1}$ Department of Biomedical Engineering, The Pennsylvania State University, University Park, PA, USA

${ }^{2}$ Center for Theoretical Biological Physics, Rice University, Houston, TX, USA

${ }^{3}$ Center for Theoretical Biological Physics, Department of Physics, and Department of Bioengineering, Northeastern University, Boston, MA, USA

${ }^{4}$ Department of Physics and Astronomy, Department of Chemistry and Department of Biosciences, Rice University, Houston, TX, USA

${ }^{5}$ Centre for BioSystems Science and Engineering, Indian Institute of Science, Bangalore, India

${ }^{6}$ Department of Mechanical Engineering and Department of Surgery, The Pennsylvania State University, University Park, PA, USA

"These authors contributed equally to this work

\#Present address: NSF-Simons Center for Multiscale Cell Fate Research, University of California, Irvine, Irvine, CA, USA

*To whom correspondence should be addressed: h.levine@northeastern.edu, jonuchic@rice.edu, mkjolly@iisc.ac.in, pak@engr.psu.edu

Keywords: Epithelial-Mesenchymal Transition, collective invasion, Notch signaling, leader cell 


\begin{abstract}
Hybrid epithelial/mesenchymal cells (E/M) are key players in aggressive cancer metastasis. A challenge is to understand how these cells, which are mostly non-existent in healthy tissue, become stable phenotypes participating collective cancer migration. The transcription factor Nrf2, which is associated with tumor progression and resistance to therapy, appears to be central to this process. Here, using a combined experimental-computational approach, we show that Nrf2 functions as a phenotypic stability factor for hybrid E/M cells by inhibiting a complete epithelial-mesenchymal transition (EMT) during collective cancer migration. We demonstrate that Nrf2 and EMT signaling are spatially coordinated near the migrating front. Computational analysis of Nrf2-EMT-Notch network and experimental modulation of Nrf2 by pharmacological treatment and CRISPR/Cas9 gene editing reveal that Nrf2 stabilizes a hybrid E/M phenotype maximally observed in the interior region immediately behind the leading edge. We further demonstrate that the Nrf2EMT-Notch network enhances DII4 and Jagged1 expression near the leading edge, which correlates with the formation of protruding tips and leader cells. Together, Nrf2 acts as a phenotypic stability factor in restricting complete EMT and coordinating collective cancer migration.
\end{abstract}




\section{INTRODUCTION}

A most devastating feature of cancer is its ability to migrate and invade adjacent tissues. ${ }^{1,2}$ During the invasion of carcinomas, cancer cells can undergo an epithelial-mesenchymal transition (EMT) to gain mesenchymal traits, such as increased motility and invasiveness. ${ }^{3}$ Emerging evidence reveals that EMT is not necessarily an irreversible, binary process; in contrast, EMT is a reversible transition process with one or multiple hybrid, or partial, epithelial/mesenchymal (E/M) states which help coordinate the collective invasion of cancer cells. ${ }^{4-6}$ These intermediate states can arise due to genetic circuits encompassing mutually inhibiting microRNA and EMT transcription factors. ${ }^{7,8}$ Hybrid E/M phenotypes have been associated with more stem cell-like traits, which include resistance to treatment and enhanced aggressiveness in comparison with a purely mesenchymal or epithelial phenotype. ${ }^{9,10}$ The clinical significance of the hybrid E/M phenotype in collective cancer invasion is evidenced by analyses of circulating tumor cell clusters with both mesenchymal and epithelial phenotypes. ${ }^{11-13}$ Furthermore, the hybrid E/M phenotype is associated with increased metastatic potential and poor clinical outcomes. ${ }^{14,15}$

EMT is a complex process involving various signaling pathways to coordinate cell-cell and cell-matrix interactions. Recent mathematical modeling and experimental analyses have demonstrated that a set of phenotypic stability factors (PSFs) can promote and stabilize hybrid E/M state(s). ${ }^{16,17}$ For instance, the transcription factor nuclear factor erythroid 2-related factor 2 (NFE2L2), commonly referred to as Nrf2, is implicated in EMT regulation ${ }^{3}$ and is associated with poor clinical outcomes in cancer patients. ${ }^{18}$ Downregulating Nrf2 in cancer cells destabilizes the hybrid E/M state and prevents collective migration while Nrf2 upregulation enriches the hybrid E/M phenotype and enables cells to migrate collectively. ${ }^{18}$ In addition, Notch signaling has also been separately implicated both in (1) the regulation of EMT and stabilization of the hybrid E/M phenotype as well as (2) in the organization of leader and follower cells at the front of collectively migrating cell layers. ${ }^{19}$ Notch is an evolutionarily conserved mechanism, which regulates cell-fate 
differentiation and cell-cell coordination. ${ }^{20,21}$ When activated in cancer cells, members of the Notch family, such as Notch1 and its ligands DII4 and Jagged1, are linked to proliferation, survival, and leader cell formation. ${ }^{19,20,22}$ Nevertheless, the complex interplays between Nrf2, EMT, and Notch signaling and their roles in collective cancer migration have not been elucidated.

In this study, we investigate the influence of Nrf2 on collective migration of cancer cells using a combined experimental-computational approach. We experimentally characterized Nrf2 and the EMT related markers, E-cadherin and ZEB1, during collective migration of urothelial bladder cancer cells. The expression of Nrf2 was modulated by a potent Nrf2 activator, sulforaphane (SFN), or by CRISPR-Cas9 gene editing. The influence of Nrf2 on EMT and Notch signaling was also investigated by a computational model of the Nrf2-EMT-Notch signaling circuit. Specifically, we coupled the intracellular Nrf2-EMT circuitry with the Notch cell-cell communication pathway, which consists of the Notch transmembrane receptor, the Notch intracellular domain (NICD), and the Notch ligands DII4 and Jagged1 in a multicellular lattice model that captures the main features of wound-induced collective cell migration. We also studied the cell migration speed and the morphology of the migrating edge in relationship to Nrf2 activation. Members of the Notch family, including Notch1, Jagged1, and DII4, were measured at the protein and/or mRNA level and the formation of leader cells and their relationship with Notch signaling was examined. The results reveal the function of Nrf2 in coordinating EMT in cancer cells during collective migration.

\section{RESULTS}

\section{Nrf2 modulates EMT during collective cell migration}

We first evaluated the relationship between Nrf2 and EMT during the collective migration of cancer cells. Previous investigations in static cell monolayers suggest that Nrf2 upregulation enhances the expression of both epithelial and mesenchymal markers (e.g., E-cadherin and ZEB1) while Nrf2 downregulation results in the attenuation of both markers. ${ }^{18}$ To study the relationship between EMT and Nrf2 in migrating monolayers, 
we performed a scratch wound healing assay over 24 hours. Cells were then fixated and fluorescently labeled for Nrf2, E-cadherin, and ZEB1 (Figure $1 \mathrm{~A}-\mathrm{C}$ ). The resulting images were then segmented into single cells for further analysis. Data were normalized to WT for each gene (Figure 1 D-F). The complete cell array was first analyzed to obtain an average intensity over the migrating monolayer (Figure 1D). Then, data were separated into cell layers to study the spatial distribution near the leading edge (Figure 1E). The intensity distribution at the leading edge was further analyzed at the single cell level (Figure 1F).

Nrf2 upregulation via sulforaphane (SFN) treatment and Nrf2 downregulation in CRISPR-Cas9 RT4-KO cells resulted in significant enhancement and reduction of Nrf2 across the migrating monolayer, respectively (Figure 1D, left column). Moreover, Nrf2 distribution showed a significant dependence on the distance from the migrating front. Sulforaphane treatment enhanced the overall level of $\mathrm{Nrf2}$, as expected, but also shifted the maximum level of Nrf2 toward the interior region (layers 3-7) of the migrating cell monolayer. In contrast, Nrf2 KO resulted in a significant decline in the Nrf2 activity, especially in the interior region of the migrating monolayer (Figure 1E, left column). We observed no significant difference across groups at the leading edge (Figure 1F, left column).

We further examined the EMT markers in the migrating monolayer. In the WT case, a reduction of E-cadherin was observed near the leading edge, suggesting that cells at the migrating front may undergo EMT, reminiscent of earlier observations. ${ }^{25}$ For the KO group, we observed an overall reduction of E-cadherin and an enhancement of ZEB1, thus suggesting that cells displayed a more mesenchymal phenotype as compared to WT. The level of ZEB1 was maximally expressed near the leading edge. Therefore, both for WT (control) and Nrf2 KO exhibited EMT activation that gradually fades as a function of distance from the leading edge. In contrast, the sulforaphane group showed high E-cadherin and ZEB1 values compared to the WT control and thus displayed a hybrid E/M phenotype (Figure 1D, center and right columns). Furthermore, when examining the spatial distribution, E-cadherin was lowest near the wound edge across all groups, and ZEB1 was highest 
near the wound edge for the KO group. Interestingly, both E-cadherin and ZEB1 were maximized at rows 3-7 for the sulforaphane group (Figure 1 E-F, center and right columns). The formation of hybrid E/M cell was further analyzed by estimating the intensity product of E-cadherin and ZEB1 (Supplementary Figure S1). The intensity product, which signifies cells with both mesenchymal and epithelial signatures, was maximized in the interior region ( row 5 ) of the migrating monolayer for WT and sulforaphane. This value was enhanced with sulforaphane treatment, and the peak shifted toward the leading edge. These results suggested that Nrf2 prevents a complete EMT and instead stabilizes a hybrid E/M cell phenotype near but not directly at the leading edge during collective cancer migration.

\section{In silico modeling predicts Nrf2-dependent increase of the hybrid E/M cell population near the leading edge}

To gain further insight into the role of Nrf2 in regulating EMT, we turned to in silico modeling of the underlying regulatory dynamics. We have previously developed a theoretical framework to describe the dynamics of EMT and Notch signaling in a 2D cell layer. ${ }^{18,26}$ Here, we generalized this framework to investigate the coupled dynamics between Nrf2, EMT, and Notch signaling. In the computational model, cells were exposed to an EMT-inducing signal that propagated throughout the cell layer, thus mimicking the spatial-dependent cellular response to the presence of the wound edge (Figure $\mathbf{2 A}$, left). The biochemical dynamics within each cell was described by interconnected feedbacks between the Nrf2, EMT, and Notch signaling modules. Moreover, binding between Notch1 and its ligands (DII4 and Jagged1) enabled communication between the biochemical circuits of neighboring cells (Figure 2A, right).

By treating the production rate of Nrf2 as a controllable parameter, we investigated the cell layer's response to varying levels of $\mathrm{Nrf2}$ induction. Specifically, we categorized cells as epithelial, hybrid E/M, or mesenchymal based on their decreasing levels of miR-200. ${ }^{24}$ Starting from randomized initial conditions, the populations of epithelial, hybrid E/M, or mesenchymal cells evolve in time depending on the level of Nrf2 induction and distance from the wound edge. At the basal, or medium, level of Nrf2, a progression of mesenchymal, hybrid 
$\mathrm{E} / \mathrm{M}$, and epithelial cells away from the edge was seen in accordance with the experiment (Figure 2D and Supplementary Movie 1). In detail, the leading edge was almost exclusively composed of mesenchymal cells, and the cells gradually transited from mesenchymal to hybrid $\mathrm{E} / \mathrm{M}$ and epithelial phenotypes toward the interior region of the migrating monolayer. The 'crossover point', where the hybrid E/M cell fraction becomes larger than the mesenchymal cell fraction, is found at a distance of about 30 cell layers from the invasive edge (Figure 2E). A weaker Nrf2 induction increased the mesenchymal cell population at the migration front while restricting the hybrid E/M cell population to the more interior region of the monolayer (Figure 2B-C and Supplementary Movie 2). Furthermore, the epithelial phenotype was almost completely suppressed. Therefore, a change from medium to weak Nrf2 induction in the computational model recapitulates many of the experimental findings seen when comparing the WT with Nrf2 KO cases. In contrast, for a strong Nrf2 induction, mesenchymal cells were observed only in the very first layers, while the hybrid $\mathrm{E} / \mathrm{M}$ cell population moved further toward the leading edge, and the crossover point occurs less than 20 cell layers from the leading edge, in excellent agreement with the experimental observation in the sulforaphane case (Figure 2FG). Overall, the computational model predicts a major role for the hybrid E/M phenotype near the leading edge under strong Nrf2 induction, in good agreement with high co-expression of epithelial and mesenchymal markers observed under SFN treatment. In particular, the coupled dynamics between Nrf2, EMT and Notch signaling drives Nrf2 to prevent a complete EMT, thus increasing the population of hybrid E/M cells near the wound edge.

Taken together, the immunochemistry data and computational predictions suggest a shift from mesenchymal to hybrid E/M cell composition toward the leading edge that depends on Nrf2 induction. This then confirms the role of Nrf2 as a "phenotypic stability factor" (PSF) that stabilizes a hybrid E/M phenotype, as previously reported in RT4 monolayers. ${ }^{18}$ Therefore, we next seek whether this transition is reflected at the level of cell migration phenotypes. 


\section{Nrf2 regulates collective cell migration}

We analyzed how Nrf2 affects the collective migration of cancer cells. The migration of the scratch wound was measured at 0 and 48 hours for all cases (Figure $3 A$ ). We observed a decrease in migration speed in both the KO and sulforaphane cases (Figure 3B). Specifically, the WT case was significantly faster than the KO and sulforaphane cases $\left(p<0.0001, n>40\right.$ cases). In accordance with other collective migration studies, ${ }^{19,27-29}$ "protruding tips" were formed at the leading edge (Figure 3C). The protruding tips often consisted 10-20 cells extended beyond the wound boundary, resulting in an irregular wound edge. These protruding tips were most profound in the WT case. The KO and SFN cases, in contrast, displayed relatively uniform wound boundary and had few or smaller protruding tips (Figure 3D). Therefore, Nrf2 may regulate collective migration by modulating the cells near the leading edge.

\section{Nrf2 modulates Notch signaling near the leading edge}

Notch signaling has been shown to control collective cell migration. ${ }^{19,22,23,30-33}$ We therefore measured the distributions of Notch markers (i.e., Notch1, Dll4, and Jagged1) in the WT and under Nrf2 perturbations (Figure 4A-C). In agreement with previous studies, ${ }^{19,23}$ Notch signaling was upregulated near the leading edge. Spatial gradients of Notch1, DII4, and Jagged1 near the leading edge were observed, and the expression levels were dependent on Nrf2. In particular, Jagged1 was negatively correlated with Nrf2 levels, being consistently highest for the KO case and lowest on the sulforaphane case in the entire monolayer (Figure 4 D-F, left column). Notch1 and Dll4 showed a mutually exclusive behavior but did not respond proportionally to Nrf2 induction. Notch1 was lowest for the WT case and higher for both KO and sulforaphane while DII4 was highest for the WT case and lowest for KO and sulforaphane cases (Figure 4D, center and right column). This behavior was especially apparent when inspecting cells near the leading edge (e.g., the first 5 rows), where we noticed a great degree of separation between WT and the other two cases (Figure 4E, center and right column). At the leading edge, Notch1 showed lowest intensity values in the WT case whereas DII4 showed the highest intensity values for the WT case (Figure 4F, center and right column). 
DII4 mRNA was reported to be upregulated in leader cells during collective migration. ${ }^{19,29}$ Particularly, mRNA levels of DII4 are more specific to leader cells and exhibit a higher contrast between leader and follower cells when compared to protein levels. ${ }^{19}$ We, therefore, evaluated the influence of Nrf2 activation on the expression of Notch1 mRNA and DII4 mRNA. We also measured the miR-200c-3p, which is a key component of the regulatory circuit driving hybrid $\mathrm{E} / \mathrm{M}$ and can attenuate Jagged1. ${ }^{34}$ Specifically, we used a doublestranded single cell biosensor as well as the FISH assay to measure the expression levels of miR-200c-3p, Notch1, and DII4 near the leading edge (Figure 5 and Supplementary Figure S2). Biosensors were added prior to the wound healing assay to ensure uniform probe internalization. ${ }^{23,35}$ Images of live migrating cells were acquired 24 hours post wound scratch to characterize the gene expression. Left panel shows fluorescence images for WT and sulforaphane cases measuring miR-200c, Notch1, and DII4 with the single cell biosensor (Figure 5A-C, left panel) and in the FISH assay (Figure 5D-F, left panel). The right panel indicates the intensity distribution as a function of distance from the migrating front and a representative distribution at the leading edge (Figure 5A-F, right panel).

A gradient of miR-200c-3p was observed near the migrating front. The level of miR-200c-3p was lowest at the leading edge and increased toward the interior region, consistent with the spatial gradient observed in Ecadherin immunostaining. Furthermore, Nrf2 activation by sulforaphane treatment enhanced the level of miR-200c-3p corresponding to an increase in epithelial and hybrid E/M cells. The gradient of miR-200c-3p and the influence of Nrf2 activation were in good agreement with the predictions of the computational model. Furthermore, Nrf2 activation suppressed the average level of DII4 and enhanced Notch1, similar to the immunocytochemistry analysis. Notably, non-uniform distributions of Dll4 were observed at the leading edge, especially for the WT case. In particular, a small number of cells at the leading edge displayed a high level of DII4. 


\section{Computational modeling predicts a transition in EMT phenotype composition and Notch signaling mode at}

\section{the leading edge due to Nrf2 increase}

Next, we returned to the mathematical model to investigate whether the response of Notch1, DII4 and Jagged1 to Nrf2 modulation could be understood in terms of the interconnected feedbacks between the Notch1 and Nrf2 pathways. Since we were especially interested in Nrf2's role in mediating collective migration and leader cell formation, we focused our analysis on the leading edge of the multicell model that can be directly compared to the front of migrating monolayer (Figure 6A and Supplementary Figure S3A-D). As Nrf2 induction increased, the molecular composition of the leading edge changed substantially. Nrf2 induction increased the number of cells with high levels of miR-200 and Notch1 (Figure 6B). The increase of Notch1 can be understood by the mutual positive feedback between NICD, Nrf2, and the Notch receptor (see circuit in Figure 2A). Notably, low-Notch cells were still observed for high Nrf2 induction levels due to lateral inhibition promoted by Notch1-DII4 signaling. Nrf2 induction also decreased the frequency of cells with high DII4 and high Jagged1 (Figure 6B). In the case of low Nrf2 induction, most cells either expressed high Dll4 or high Jagged1. A small fraction of cells, however, co-expressed both ligands, reflective of a hybrid Sender/Receiver phenotype enabled by a balance of lateral inhibition and lateral induction (Figure 6C). ${ }^{20}$ This effect was progressively removed by a stronger Nrf2 induction, as cells expressing either one or both ligands become rarer (Supplementary Figure S3E).

In terms of EMT phenotype composition, the invading edge was predominantly composed of mesenchymal cells when Nrf2 induction is low. Conversely, at higher Nrf2 levels, the invasive edge was a mixture of hybrid E/M and epithelial cells (Figure 6D). More generally, Nrf2 induction correlated with an average increase in miR-200 and Notch1 expression at the invasive edge, as well as decrease of DII4 and Jagged1 expression, similar to the trend observed in the experiments. Therefore, the model predicts that Nrf2 induces a transition from a mostly mesenchymal invading edge with strong DII4 and Jagged1 signaling to a mostly hybrid E/M and 
epithelial invasive edge with high Notch1 expression, in good agreement with the trend observed experimentally when increasing Nrf2 activation from WT to SFN.

\section{Leader cell formation is optimal for the WT case and DII4 is highest near the wound edge}

The formation of protruding tips and modulation of the Notch ligand DII4 suggest Nrf2 may control collective cancer migration by regulating the formation of leader cells. We thus investigated leader cells at the wound edge (Figure 7). In this study, we defined leader cells based on their spatial location at the protruding tips and their interactions with follower cells. Bright-field images at the leading edge also revealed distinct morphologies of leader cells for KO, WT, and sulforaphane cases (Figure 7A). The leader cells in the WT case showed aggressive morphologies, including enlarged cell size and active lamellipodial structures, and entrained more follower cells when compared with leader cells in KO and sulforaphane cases. The WT case also exhibited the highest density of leader cells when compared to the other cases, which correlated with the formation of protruding tips (Figure 7B).

We further analyzed molecular markers of leader cells in the WT case at the protein and mRNA levels (Figure 7C-H). Leader cells generally showed a low level of E-cadherin and a high level of ZEB1. This is expected as most cells at the leading edge exhibit a mesenchymal phenotype. Leader cells also expressed a low level of Notch1 while upregulating both Jagged1 and DII4. This observation is particularly interesting as DII4 and Jagged 1 are often assumed as mutually exclusive Notch signaling states, ${ }^{36,37}$ and confirms the model's prediction that a population of high DII4 and high Jagged1 cells exists at the leading edge due to biochemical feedbacks between the Notch, EMT and Nrf2 signaling modules. Crucially, Jagged1 was relatively uniform in all cells at the leading edge while DII4 was selectively upregulated in leader cells (Figure $\mathbf{7 H}$ ). The selective upregulation of DII4 in leader cells was particularly profound at the mRNA level (Figure $\mathbf{7 1 , K}$ ), which is consistent with previous leader cell investigation, where DII4 was distinctively upregulated in leader cells at the mRNA level. ${ }^{19}$ In turn, Notch1 was dramatically downregulated in leader cells while being upregulated in 
follower cells (Figure $7 \mathrm{~J}, \mathrm{~L}$ ). This is in agreement with model predictions indicating the mutually exclusive states for DII4 and Notch1.

\section{DISCUSSION}

This study investigated the role of Nrf2 in regulating the collective migration of cancer cells. While Nrf2 has been widely studied in the context of antioxidant response and chemoresistance, ${ }^{38-41}$ its function in cancer progression and invasion remains poorly understood.,.$^{3,18,42-44}$ Our experimental-computational analysis revealed that the Nrf2-EMT-Notch network coordinates cancer cells near the leading edge during collective migration. In particular, Nrf2 acted as a PSF in suppressing a full EMT and promoting a hybrid E/M phenotype, in a spatially coordinated manner. In the unperturbed condition (i.e., WT), the cells near the migrating front displayed a gradient of epithelial to mesenchymal behaviors. The cells at the leading edge were relatively mesenchymal while the cells in the monolayer displayed an epithelial phenotype. It should be noted that cells at the leading edge maintained cell-cell contact with neighboring cells and expressed a detectable level of Ecadherin, supporting a partial EMT identification instead of a full EMT. In both experimental and computational models, Nrf2 promoted the hybrid E/M cells, which were positioned at an interior region behind the leading edge. Nrf2 activation was required to maintain the hybrid E/M state of these cells. As indicated in $\mathrm{Nrf2} \mathrm{KO}$, the cells shifted toward a more mesenchymal state, and the level of E-cadherin expression was significantly attenuated in the migrating monolayer. In contrast, Nrf2 upregulation enhanced the hybrid E/M phenotype and increased both epithelial and mesenchymal markers, especially in the interior region (several rows behind the leading edge) in the migrating monolayer.

Our results shed light on the involvement of Notch signaling in collective cancer migration. Notch signaling regulates cell-cell coordination during development and has been implicated in cancer progression. ${ }^{19,20}$ Our results suggest that the Nrf2-EMT-Notch network leads to spatial coordination of cancer cells. In particular, cancer cells several rows behind the leading edge exhibited upregulated Notch1, Nrf2, and miR-200c while 
cells at the leading edge expressed ZEB1, DII4, and Jagged1. The spatial coordination is contributed by the elevated Nrf2-Notch-NICD activity in the interior region and enhanced ZEB1, which reduced miR-200c ${ }^{45}$ and consequently increased Jagged1, at the leading edge. The Nrf2-EMT-Notch network promoted the upregulation of DII4 and Jagged1 at the leading edge, which appeared to correlate with the formation of leader cells and protrusion tips. DII4 is associated with the formation of leader cells during collective cancer invasion. ${ }^{19,22,29}$ Jagged1 is also shown to promote MYO10 driven filopodial persistence for fibronectin micropatterning of leader cells. ${ }^{46}$ Our computational analysis shows that the coordination between DII4 and Jagged1 is highly sensitive to Nrf2 activation. Our predictions indicate that cells expressing both Dll4 and Jagged1 or having mutually exclusive behaviors could exist at the leading edge (Figure 6C). Therefore, our results suggest Nrf2 coordinates DII4 and Jagged1 near the leading edge to regulate different aspects of leader cells. Similar hypotheses have been drawn in contexts of inner ear development and sprouting angiogenesis, where a weak Jagged1 signaling was proposed to further amplify Notch1-DIl4 lateral inhibition by further sequestering Notch1 ligands in high Notch1 receiver cells. ${ }^{37,47}$ Moreover, Jagged1 can: (1) induce partial EMT and cancer stem cell traits and (2) propagate these aggressive traits to neighboring cells via Notch1-Jagged1 signaling. ${ }^{14,37,47}$ Therefore, the precise dynamics acting between DII4 and Jagged1 and its functional implications in cancer invasion should be further investigated.

Our data indicate that Nrf2 modulates the migration speed of collective cancer migration. Cancer cells, at least in our model of urothelial bladder cancer, have their maximum migration speed in the unperturbed condition. Previous studies report both positive and negative effects of Nrf2 on the collective invasion of various cancer cell types. ${ }^{42,44,48-51}$ In general, cancer cell migration can be influenced by multiple factors, such as leader cell formation, cell motility, and proliferation. In our experiment, the migration speed correlated with DII4 expression and the formation of protruding tips and leader cells. Furthermore, EMT is associated with the motility and proliferation of cancer cells. ${ }^{52,53}$ We show that Nrf2 activation by sulforaphane treatment promoted the hybrid E/M phenotype and enhanced proliferation (Supplementary Figure S4). All these factors 
can contribute to the migratory behavior, and the overall effect of Nrf2 on collective cancer migration may be context specific. Furthermore, additional signaling pathways and molecular programs, such as stemness and metabolic switching, ${ }^{54-56}$ may also be involved in the regulation of the overall cancer invasion process. The complex interplay between signaling and migratory dynamics also underscores future theoretical challenges that are not explicitly considered in our current model, including (1) the coupling of biochemical and mechanical regulation of cell migration, (2) the effect of cell proliferation on cell patterning, and (3) the context-specificity of the EMT program in terms of both transcriptional response and number of intermediate phenotypes in the EMT spectrum. ${ }^{57-59}$ Future experimental and computational investigations will be required to fully understand the role of Nrf2 on cancer invasion, and more physiologically relevant invasion models should be investigated to understand the impact of the Nrf2-EMT-Notch network on collective cancer invasion.

\section{MATERIALS AND METHODS}

\section{Cell culture and reagents}

The human RT4 cells (labeled as WT) were purchased from American Type Culture Collection (ATCC, USA). The CRISPR-Cas9 knockout cell pool RT4-Nrf2-KO (labeled as KO) was obtained from Synthego, CA. DLsulforaphane (cat. \#s4441, Sigma Aldrich, USA) was dissolved in DMSO (cat. \#D8418, Sigma Aldrich, USA) according to manufacturer's instructions. DL-sulforaphane was added to the RT4 cells (labeled as SFN) at a final concentration of $7.5 \mu \mathrm{M}$ immediately after the wound scratch assay. All cells were cultured in McCoy's 5A medium containing 10\% FBS and 0.1\% Gentamicin (Fisher Scientific, Hampton, NH). Cells were maintained at $37^{\circ} \mathrm{C}$ in $5 \% \mathrm{CO}_{2}$, and media was refreshed every 2 days. The following gRNA targeting exon 2 was used for NFE2L2-KO: AUUUGAUUGACAUACUUUGG. Knockout cells show a predicted functional knockout of $63 \%$ which was confirmed by Synthego through RT-qPCR showing 75\% editing efficiency post expansion at passage 4. All experiments were done between passages 5-8 for the CRISPR/Cas9 Nrf2-KO cells. All experiments were performed in polystyrene 24-well plates (cat. \# 07-200-740, Fisher Scientific, Hampton, NH). 
Double-stranded locked nucleic acid (dsLNA) biosensors and synthetic targets for calibration were synthesized by Integrated DNA Technologies (San Diego, CA). The sequences are available in Supplementary Table 1. The following reagents were used to perform fluorescence in-situ hybridization (FISH): Stellaris RNA FISH Hybridization Buffer (cat. \#SMF-HB1-10), Stellaris RNA FISH Wash Buffer A (cat. \#SMF-WA1-60), and Stellaris RNA FISH Wash Buffer B (cat. \#SMF-WB1-20). All FISH reagents were acquired from Biosearch Technologies. Transfection reagents for the dsLNA biosensors were acquired from Thermofisher scientific, unless specified otherwise.

\section{Immunocytochemistry}

Cells were washed with warmed 1x PBS twice, followed by fixation with chilled $4 \%$ paraformaldehyde (Sigma) in PBS for 15 minutes, all reagents were kept cold past this point and incubation was performed at room temperature unless stated otherwise. Permeabilization was performed with $1 \%$ Triton X-100 in PBS for 10 minutes followed by a blocking step with $3 \%$ bovine serum albumin (BSA) in PBS for 30 minutes. The cells were incubated overnight at $4^{\circ} \mathrm{C}$ with the primary antibodies and then incubated in the dark for 2 hours against the secondary antibodies. Primary antibodies used were Nrf2 (1:100, cat. \#AF3925, R\&D Biosystems), E-cadherin (1:50, cat. \#M3612, Agilent Dako), ZEB1 (1:100, cat. \#ab124512, Abcam), Jagged1 (1:50, cat. \#sc390177, Santa Cruz Biotechnology), Notch1 (1:100, cat. \#ab8925, Abcam), DIl4 (1:100, cat. \#PA585931, Thermofisher Scientific). Secondary antibodies used were Alexa-fluor conjugated secondary antibodies (1:1000, Life technologies). The antibodies were all diluted in 3\% BSA solution. Wells were washed 3 times with PBS in between each step. Cells were examined using a laser scanning confocal microscope (Leica TCS SP8; Leica Microsystems, Wetzlar, Germany) immediately after the last washing step.

\section{Wound-Healing Assay}


The scratch wound healing assay was performed to determine cell migration using confluent cultures. Briefly, at $100 \%$ confluency, the monolayer was scratched with a sterile $200 \mu \mathrm{L}$ pipet tip and media was refreshed for all wells. Cells were washed with warm $1 x$ PBS before and after wounding. Images were acquired at 0, 24 and 48 hours. The experiments were repeated at least 3 times and the mean and standard deviation were calculated using the freeware Image-J. Cell migration was expressed as the migration rate in microns per hour $(\mu \mathrm{m} / \mathrm{h})$ : (original scratch width - final scratch width)/time.

\section{Single cell gene expression analysis and transfection}

The dsLNA biosensors were used to measure mRNA and microRNA (miRNA) expression profiles of target genes near the migrating front of the monolayer. The design, characterization, and protocol were described previously. ${ }^{19,23}$ Briefly, the complementary sequence to the target mRNA/miRNA (labeled as probe) is labeled with a fluorophore at the $5^{\prime}$ end. A complementary sequence with a quencher at the $3^{\prime}$ end (labeled as quencher) is designed. All sequences were verified through the NCBI Basic Local Alignment Search Tool for nucleotides (BLASTn). A random probe with no known intracellular targets was also developed as a negative control. For transfection, the probe and quencher were dissolved in $10 \mathrm{mM}$ Tris-EDTA buffer and $0.2 \mathrm{M} \mathrm{NaCl}$ before mixing at a 1:2.5 ratio. Then, the probe and Lipofectamine RNAiMAX reagent (cat. \#13778075, Thermofisher) were diluted in Opti-MEM media (cat. \#31985062, Thermofisher) according to the manufacturer's protocol. Cells were seeded in a 24 -well plate and transfected once they reached $90-95 \%$ confluency. Each well contained a total of $1 \mu \mathrm{g}$ probe with $2 \mu \mathrm{L}$ Lipofectamine RNAiMAX. The dsLNA biosensors targeting different genes were transfected in separate wells.

\section{Fluorescent in situ hybridization assay}

FISH was used to measure mRNA and miRNA expression of target genes in fixed cells near the wound boundary. The FISH assay was performed according to manufacturer's instructions with the probes designed for the single-cell biosensors. Briefly, 24 hours after the wound scratch assay, the cells were fixed using $3.7 \%$ 
formaldehyde in 1x PBS and incubated at room temperature for 10 minutes. Cells were then washed twice with 1x PBS and permeabilized using 70\% ethanol in deionized (DI) water for at least 1 hour at $4{ }^{\circ} \mathrm{C}$. Afterwards, cells were washed with Wash Buffer A (cat. \#SMF-WA1-60, Biosearch technologies) for 5 minutes. Then the miR-200c-3p, DII4 mRNA and Notch1 mRNA probes were mixed with the hybridization buffer (cat. \# SMFHB1-10, Biosearch technologies) according to the manufacturer's instructions, covered in foil and placed in the cell incubator at $37^{\circ} \mathrm{C}$ for 5 hours, all subsequent steps were performed in the dark. Then, cells were incubated in Wash Buffer A for 30 minutes and placed in the incubator. Lastly, cells were incubated with Wash Buffer B (cat. \# SMF-WB1-20, Biosearch technologies) for 5 minutes. Wells were replenished with fresh 1x PBS and imaged immediately using a laser scanning confocal microscope (Leica TCS SP8; Leica Microsystems, Wetzlar, Germany).

\section{Imaging and data analysis}

All images were acquired using a laser scanning confocal microscope (Leica TCS SP8; Leica Microsystems, Wetzlar, Germany). Wound healing migration data was analyzed in ImageJ. For all other experiments, the bright-field image was used as a template to create a mask to segment the images into single cells using Adobe Photoshop. The segmented images were then transferred to MATLAB for a complete analysis of morphological and fluorescence features at a single-cell level.

\section{Leader cell selection and quantification}

In this study, leader cells in the migrating monolayer are defined as cells at the migrating tip with apparent cell-cell contact with follower cells behind them. To be classified as a leader cell, we considered the distance from the initial wound boundary, the extent of the migration sprout created by the leader cell, and the contact with follower cells. To quantify number of leader cells per case (i.e., KO, WT, and SFN), the number of leader cells was counted per migration edge per case. The value was reported as leaders/mm, that is: (total \# of leader cells / $1 \mathrm{~mm}$ wound edge). 


\section{Statistical analysis}

Data obtained from MATLAB and ImageJ were analyzed using the statistical software GraphPad Prism 9. Experiments measuring mRNA/microRNA levels were performed at least 3 times in multiple experiments. All other assays were performed at least 4 times in multiple experiments. In single-cell measurement experiments, at least 500 cells per case were analyzed. For first cell layer (i.e., the migrating edge) analyses, at least 100 cells per case were measured. All datasets were considered to follow a non-normal distribution. Therefore, non-parametric tests were utilized to compare across groups where possible. The tests used were: Kruskal-Wallis test with the Dunn's multiple comparisons test, a Two-Way ANOVA test with a post-hoc Tukey test including multiple comparisons across rows and columns, and the ROUT method to identify outliers (Figures 3D and 7B). The following values were assigned to test for significance: $n s p$-value $>0.05, * p$-value $<$ $0.05, * * p$-value $<0.01, * * * p$-value $<0.001$, and $* * * *$-value $<0.0001$

\section{Multicell model of Nrf2-EMT-Notch signaling}

We employed a continuous mass action model to describe the biochemical interactions between molecular players in the EMT, Nrf2 and Notch circuits. Within a cell, the temporal dynamics for the copy number of any given variable (say, $X$ ) is described by the generic equation:

$$
\frac{d X}{d t}=K_{X}-\gamma_{X} X
$$

In eq. (1), the first term on the right-hand side (RHS) is a production rate with units of number of molecules produced per unit time. The effect of other microRNAs or transcription factors (TF) that induce or inhibit the production of $X$ is modeled by additional functions that modulate the basal production rate. The second term on the RHS models molecule degradation. 
The effect of transcriptional activation or inhibition exerted by a regulator (say, R) on another given species in the circuit is modeled with a shifted Hill function:

$$
H^{S}\left(R, R_{0}, n, \lambda\right)=\frac{1}{1+\left(\frac{R}{R_{0}}\right)^{n}}+\lambda \frac{\left(\frac{R}{R_{0}}\right)^{n}}{1+\left(\frac{R}{R_{0}}\right)^{n}}
$$

where $R$ is the concentration or copy number of the regulator and $R_{0}$ is the half-maximal concentration parameter expressed in same units of $R$. Additionally, the Hill coefficient $n$ describes the steepness in transcriptional response with respect to the regulator concentration. Finally, the fold change $\lambda$ describes the change in target level due to regulation by $R(\lambda<1$ implies that $R$ is an inhibitor, while $\lambda>1$ implies that $R$ is an activator). If a species is regulated by multiple TFs, Hill functions are multiplied in the production rate of eq. (1).

Moreover, microRNAs can inhibit the production of other species in the circuit by binding the target mRNA and facilitating their degradation. This post-translational inhibition is modeled following the microRNA-TF chimera toggle switch model first introduced by Lu and collaborators. ${ }^{24}$ First, a first set of functions $P_{l}(\mu, n)$ quantifies the inhibition that a microRNA $(\mu)$ exerts on the target TF; here, $n$ is the number of sites for microRNA binding to the mRNA. Furthermore, a second set of functions $P_{y}(\mu, n)$ describes the corresponding decrease of microRNA due to the degradation of the microRNA/mRNA complex.

In the multicellular model, cells are arranged on a two-dimensional hexagonal grid. The intracellular signaling dynamics of Nrf2, EMT and Notch is described within each cell by the coupled system of ODEs. Moreover, the biochemical networks of neighboring cells are coupled via ligand-receptor binding between Notch and its ligands, DII4 and Jagged1. For any given cell $(i)$ in the lattice, the numbers of external Notch receptors and ligands available for binding $\left(N_{E X T}{ }^{(i)}, D_{E X T}{ }^{(i)}, J_{E X T}{ }^{(i)}\right)$ are calculated as the sums over the cell's nearest neighbors: 


$$
\begin{aligned}
& N_{E X T}{ }^{(i)}=\sum_{j \in N(i)} N_{j} \\
& D_{E X T}{ }^{(i)}=\sum_{j \in N(i)} D_{j} \\
& J_{E X T}{ }^{(i)}=\sum_{j \in N(i)} J_{j}
\end{aligned}
$$

Moreover, to simulate the conditions of high stress close to the migrating front, we introduce a gradient of an EMT-inducing signal $(I(x, y, t))$ that is secreted at the left end of the lattice (the wound edge), diffuses along the $\mathrm{x}$-coordinate and is removed at the opposite end of the lattice. This diffusion dynamics gives rise to a profile where cells close to the invasive edge are highly exposed to EMT-inducing signaling while cells in the interior are weakly exposed. The complete set of equations for all molecular species in the Nrf2, EMT and Notch circuits, as well as parameters used can be found in the supplementary information.

\section{Competing interests}

The authors declare no competing interests.

\section{Acknowledgements}

This work was supported by National Science Foundation by sponsoring the Center for Theoretical Biological Physics - award PHY-2019745 (JNO, HL) and by awards PHY-1605817 (HL), CHE-1614101 (JNO) and CBET-1802947 (PKW). F.B. was also supported by the NSF grant DMS1763272 and a grant from the Simons Foundation (594598, QN). MKJ was supported by Ramanujan Fellowship awarded by SERB, DST, Government of India (SB/S2/RJN-049/2018). JNO is a CPRIT Scholar in Cancer Research sponsored by the Cancer Prevention and Research Institute of Texas.

\section{DATA AVAILABILITY}


bioRxiv preprint doi: https://doi.org/10.1101/2021.04.21.440858; this version posted April 22, 2021. The copyright holder for this preprint (which was not certified by peer review) is the author/funder. All rights reserved. No reuse allowed without permission.

All the data supporting the findings of this study are available within the article and its supplementary information files and from the corresponding author upon reasonable request. 


\section{References:}

1. Hanahan, D. \& Weinberg, R. A. Hallmarks of cancer: the next generation. Cell 144, 646-74 (2011).

2. Beunk, L., Brown, K., Nagtegaal, I., Friedl, P. \& Wolf, K. Cancer invasion into musculature: Mechanics, molecules and implications. Semin. Cell Dev. Biol. 93, 36-45 (2019).

3. Riahi, R. et al. Single cell gene expression analysis in injury-induced collective cell migration. Integr. Biol. (United Kingdom) 6, 192-202 (2014).

4. Brabletz, T., Kalluri, R., Nieto, M. A. \& Weinberg, R. A. EMT in cancer. Nat. Rev. Cancer 18, 128-134 (2018).

5. Nieto, M. A., Huang, R. Y. Y. J., Jackson, R. A. A. \& Thiery, J. P. P. Emt: 2016. Cell 166, 21-45 (2016).

6. Kröger, C. et al. Acquisition of a hybrid E/M state is essential for tumorigenicity of basal breast cancer cells. Proc. Natl. Acad. Sci. U. S. A. 116, 7353-7362 (2019).

7. Lu, M., Jolly, M. K., Levine, H., Onuchic, J. N. \& Ben-Jacob, E. MicroRNA-based regulation of epithelialhybrid-mesenchymal fate determination. Proc. Natl. Acad. Sci. U. S. A. 110, 18144-18149 (2013).

8. Zhang, J. et al. TGF- $\beta$-induced epithelial-to-mesenchymal transition proceeds through stepwise activation of multiple feedback loops. Sci. Signal. 7, ra91 (2014).

9. Jolly, M. K. et al. Implications of the hybrid epithelial/mesenchymal phenotype in metastasis. Front. Oncol. 5, 155 (2015).

10. Pastushenko, l. et al. Identification of the tumour transition states occurring during EMT. Nature 556, 463-468 (2018).

11. Quan, Q. et al. Cancer stem-like cells with hybrid epithelial/mesenchymal phenotype leading the collective invasion. Cancer Sci. 111, 467-476 (2020).

12. Aiello, N. M. et al. EMT Subtype Influences Epithelial Plasticity and Mode of Cell Migration. Dev. Cell 45, 681-695.e4 (2018).

13. Jolly, M. K. et al. Stability of the hybrid epithelial/mesenchymal phenotype. Oncotarget 7, 2706727084 (2016). 
14. Jia, D. et al. Quantifying Cancer Epithelial-Mesenchymal Plasticity and its Association with Stemness and Immune Response. J. Clin. Med. 8, 725 (2019).

15. Bocci, F. et al. NRF2 activates a partial epithelial-mesenchymal transition and is maximally present in a hybrid epithelial/mesenchymal phenotype. Integr. Biol. (Camb). 11, 251-263 (2019).

16. Biswas, K., Jolly, M. K. \& Ghosh, A. Stability and mean residence times for hybrid epithelial/mesenchymal phenotype. Phys. Biol. 16, (2019).

17. Bocci, F. et al. Numb prevents a complete epithelial-mesenchymal transition by modulating Notch signalling. J. R. Soc. Interface 14, 20170512 (2017).

18. Bocci, F. et al. NRF2 activates a partial epithelial-mesenchymal transition and is maximally present in a hybrid epithelial/mesenchymal phenotype. Integr. Biol. (2019). doi:10.1093/intbio/zyz021

19. Riahi, R. et al. Notch1-DIl4 signalling and mechanical force regulate leader cell formation during collective cell migration. Nat. Commun. 6, 6556 (2015).

20. Boareto, M. et al. Jagged-Delta asymmetry in Notch signaling can give rise to a Sender/Receiver hybrid phenotype. Proc. Natl. Acad. Sci. 112, E402-E409 (2015).

21. Bray, S. J. Notch signalling in context. Nat. Rev. Mol. Cell Biol. 17, 722-735 (2016).

22. Torab, P. et al. Three-Dimensional Microtumors for Probing Heterogeneity of Invasive Bladder Cancer. Anal. Chem. 92, 8768-8775 (2020).

23. Dean, Z. S., Elias, P., Jamilpour, N., Utzinger, U. \& Wong, P. K. Probing 3D collective cancer invasion using double-stranded locked nucleic acid biosensors. Anal. Chem. 88, 8902-8907 (2016).

24. Lu, M., Jolly, M. K., Levine, H., Onuchic, J. N. \& Ben-Jacob, E. MicroRNA-based regulation of epithelialhybrid-mesenchymal fate determination. Proc. Natl. Acad. Sci. 110, 18144-18149 (2013).

25. Brabletz, T. et al. Variable -catenin expression in colorectal cancers indicates tumor progression driven by the tumor environment. Proc. Natl. Acad. Sci. 98, 10356-10361 (2001).

26. Bocci, F. et al. Toward understanding cancer stem cell heterogeneity in the tumor microenvironment. Proc. Natl. Acad. Sci. U. S. A. 116, 148-157 (2019). 
27. Nagai, T., Ishikawa, T., Minami, Y. \& Nishita, M. Tactics of cancer invasion: solitary and collective invasion. J. Biochem. 167, 347-355 (2020).

28. Mayor, R. \& Etienne-Manneville, S. The front and rear of collective cell migration. Nat. Rev. Mol. Cell Biol. 17, 97-109 (2016).

29. Konen, J. et al. Image-guided genomics of phenotypically heterogeneous populations reveals vascular signalling during symbiotic collective cancer invasion. Nat. Commun. 8, 15078 (2017).

30. Xiao, Y., Riahi, R., Torab, P., Zhang, D. D. \& Wong, P. K. Collective Cell Migration in 3D Epithelial Wound Healing. ACS Nano 13, 1204-1212 (2019).

31. Jakobsson, L. et al. Endothelial cells dynamically compete for the tip cell position during angiogenic sprouting. Nat. Cell Biol. 12, 943-953 (2010).

32. Aman, A. \& Piotrowski, T. Cell-cell signaling interactions coordinate multiple cell behaviors that drive morphogenesis of the lateral line. Cell Adhes. Migr. 5, 499-508 (2011).

33. Meurette, O. \& Mehlen, P. Notch Signaling in the Tumor Microenvironment. Cancer Cell 34, 536-548 (2018).

34. Boareto, M. et al. Notch-Jagged signalling can give rise to clusters of cells exhibiting a hybrid epithelial/mesenchymal phenotype. J. R. Soc. Interface 13, 20151106 (2016).

35. Dean, Z. S., Riahi, R. \& Wong, P. K. Spatiotemporal dynamics of microRNA during epithelial collective cell migration. Biomaterials 37, 156-163 (2015).

36. Bocci, F., Onuchic, J. N. \& Jolly, M. K. Understanding the Principles of Pattern Formation Driven by Notch Signaling by Integrating Experiments and Theoretical Models. Front. Physiol. 11, 1-24 (2020).

37. Petrovic, J. et al. Ligand-dependent Notch signaling strength orchestrates lateral induction and lateral inhibition in the developing inner ear. Dev. 141, 2313-2324 (2014).

38. Kaspar, J. W., Niture, S. K. \& Jaiswal, A. K. Nrf2:INrf2 (Keap1) signaling in oxidative stress. Free Radic. Biol. Med. 47, 1304-1309 (2009).

39. LAU, A., VILLENEUVE, N., SUN, Z., WONG, P. \& ZHANG, D. Dual roles of Nrf2 in cancer. Pharmacol. 
Res. 58, 262-270 (2008).

40. Wang, X. J. et al. Nrf2 enhances resistance of cancer cells to chemotherapeutic drugs, the dark side of Nrf2. Carcinogenesis 29, 1235-1243 (2008).

41. Kamble, D., Mahajan, M., Dhat, R. \& Sitasawad, S. Keap1-Nrf2 Pathway Regulates ALDH and Contributes to Radioresistance in Breast Cancer Stem Cells. Cells 10, 1-21 (2021).

42. Pan, H. et al. The role of Nrf2 in migration and invasion of human glioma cell U251. World Neurosurg. 80, 363-370 (2013).

43. Xu, J. et al. Mild oxidative stress reduces Nrf2 sumOylation to promote Kras/LKB1/Keap1 mutant lung adenocarcinoma cell migration and invasion. Oxid. Med. Cell. Longev. 2020, (2020).

44. Ko, E., Kim, D., Min, D. W., Kwon, S. H. \& Lee, J. Y. Nrf2 regulates cell motility through RhoA-ROCK1 signalling in non-small-cell lung cancer cells. Sci. Rep. 11, 1-9 (2021).

45. Brabletz, S. et al. The ZEB1/miR-200 feedback loop controls Notch signalling in cancer cells. EMBO J. 30, 770-82 (2011).

46. Summerbell, E. R. et al. Epigenetically heterogeneous tumor cells direct collective invasion through filopodia-driven fibronectin micropatterning. Sci. Adv. 6, 1-16 (2020).

47. Kang, T. Y. et al. Pericytes enable effective angiogenesis in the presence of proinflammatory signals. Proc. Natl. Acad. Sci. U. S. A. 116, 23551-23561 (2019).

48. Zhang, Q. \& Yang, D. Allicin suppresses the migration and invasion in cervical cancer cells mainly by inhibiting NRF2. Exp. Ther. Med. 1523-1528 (2018). doi:10.3892/etm.2018.7104

49. Zhang, H. et al. Nrf2 promotes breast cancer cell migration via up-regulation of G6PD/HIF-1 $\alpha /$ Notch1 axis. J. Cell. Mol. Med. 23, 3451-3463 (2019).

50. Zhang, M. et al. Nrf2 is a potential prognostic marker and promotes proliferation and invasion in human hepatocellular carcinoma. BMC Cancer 15, 531 (2015).

51. Wang, F., Liu, P., An, H. \& Zhang, Y. Sulforaphane suppresses the viability and metastasis, and promotes the apoptosis of bladder cancer cells by inhibiting the expression of FAT-1. Int. J. Mol. 
Med. 46, 1085-1095 (2020).

52. Hecht, I. et al. The motility-proliferation-metabolism interplay during metastatic invasion. Sci. Rep. 5, $1-10$ (2015).

53. Pastushenko, I. \& Blanpain, C. EMT Transition States during Tumor Progression and Metastasis.

Trends Cell Biol. 29, 212-226 (2019).

54. Han, T. et al. How does cancer cell metabolism affect tumor migration and invasion? Cell Adhes.

Migr. 7, 395-403 (2013).

55. Jolly, M. K. et al. Implications of the hybrid epithelial/mesenchymal phenotype in metastasis. Front.

Oncol. 5, 155 (2015).

56. Commander, R. et al. Subpopulation targeting of pyruvate dehydrogenase and GLUT1 decouples metabolic heterogeneity during collective cancer cell invasion. Nat. Commun. 11, 1533 (2020).

57. Deng, Y., Chakraborty, P., Jolly, M. K. \& Levine, H. A Theoretical Approach to Coupling the EpithelialMesenchymal Transition (EMT) to Extracellular Matrix (ECM) Stiffness via LOXL2. Cancers (Basel). 13, 1609 (2021).

58. McFaline-Figueroa, J. L. et al. A pooled single-cell genetic screen identifies regulatory checkpoints in the continuum of the epithelial-to-mesenchymal transition. Nat. Genet. 51, 1389-1398 (2019).

59. Cook, D. P. \& Vanderhyden, B. C. Context specificity of the EMT transcriptional response. Nat.

Commun. 11, 2142 (2020). 
A

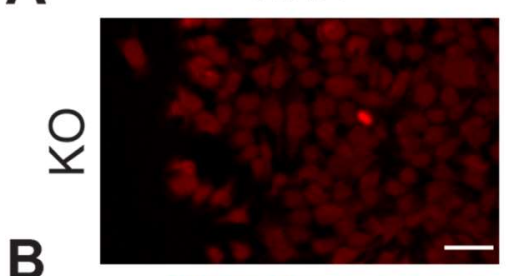

B

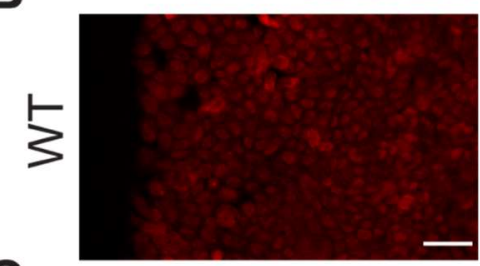

C

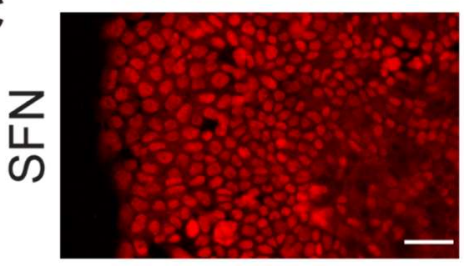

D

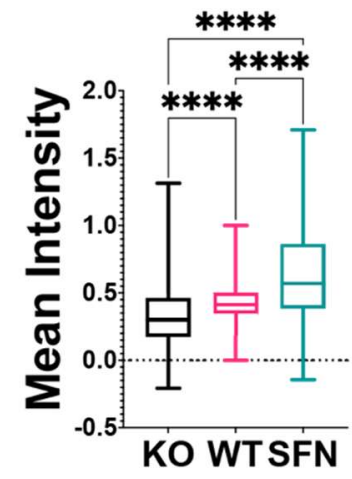

E

F
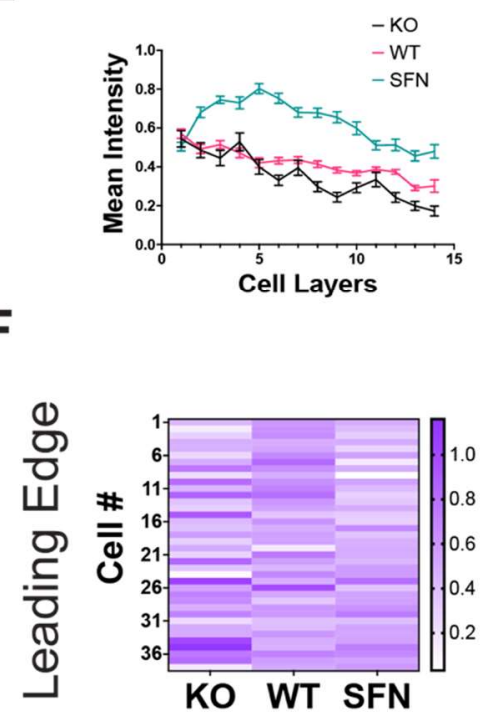

ZEB1
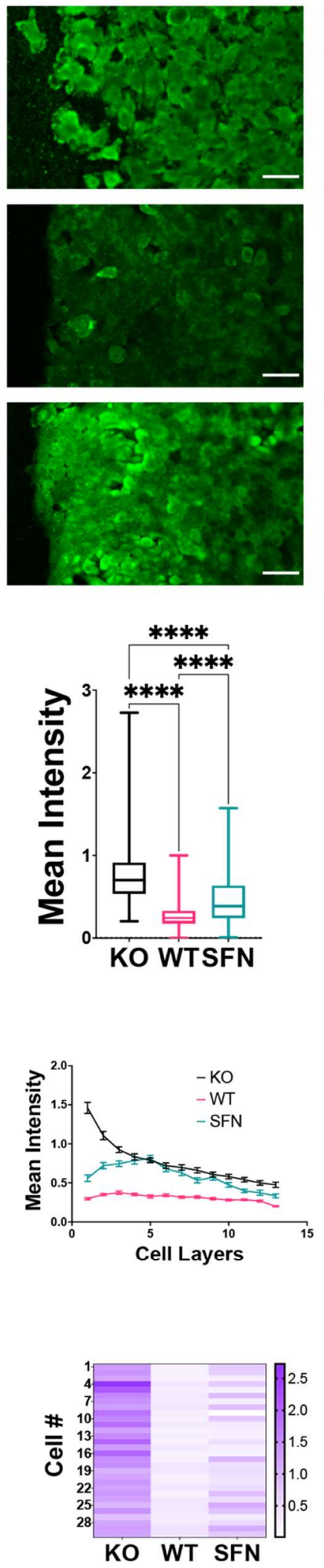

E-cadherin
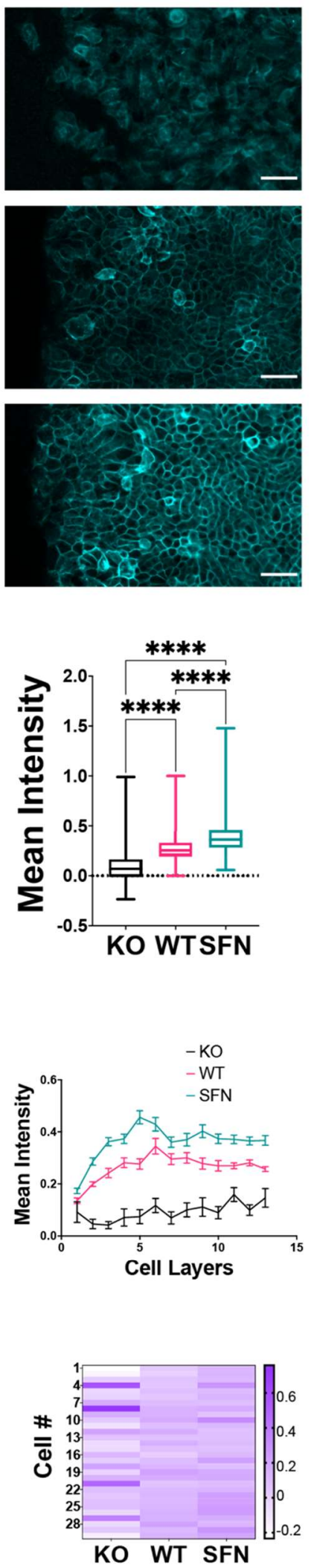
Figure 1. Nrf2 regulates EMT near the leading edge during collective migration. (A-C) Immunocytochemistry of RT4 bladder cancer cells measuring (A) Nrf2, (B) E-cadherin, and (C) ZEB1 protein levels in a wound healing assay for CRISPR/Cas9 NFE2L2-KO Pool RT4 cells (KO), wild-type RT4 cells (WT), and sulforaphane treated RT4 cells (SFN), respectively. Scale bars, $50 \mu \mathrm{m}$. (D-F) Quantification of immunocytochemistry data. (D) Average intensity over the whole cell layer, $(E)$ intensity distribution in the migrating monolayer measured as number of cell layers, and (F) heatmap of representative cells near the wound leading edge for Nrf2, E-cadherin, and ZEB1, respectively. The nonparametric Kruskal-Wallis test along with the Dunn's multiple comparisons test were used to compare across groups. For each experiment $\mathrm{n}>500$ cells per condition. ${ }^{* * * *} \mathrm{p}$-value $<0.0001$. Images are representatives from 6 experiments. 

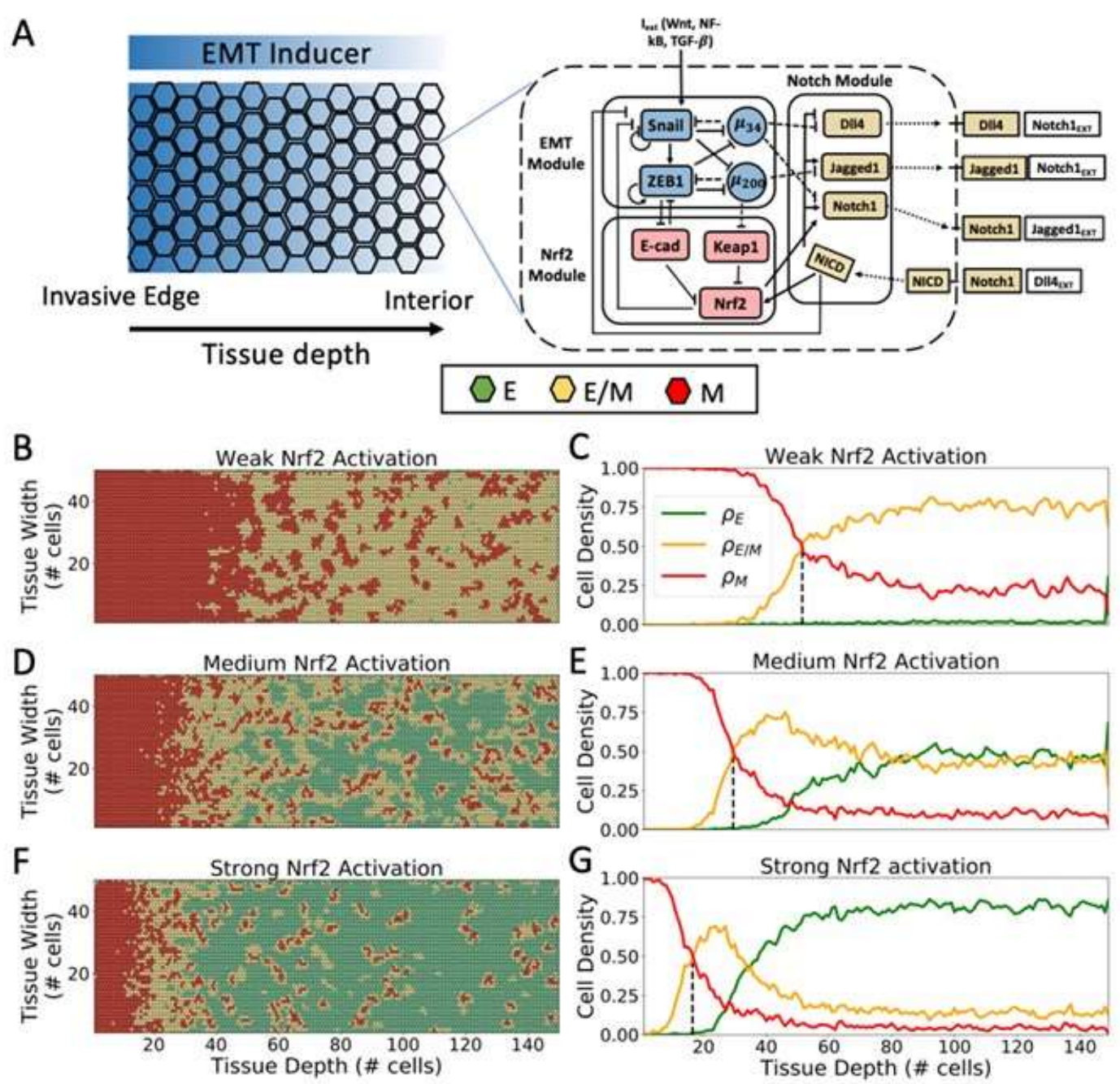

Figure 2. Spatial patterning of cells in the multicell model of wound healing. (A) Left: In the multicell model, cells are arranged on a hexagonal lattice. Cells at the leftmost region (invasive edge) are highly exposed to an external EMT-inducing signal, while cells in the interior are weakly exposed. Right: The signaling dynamics within each cell is described by the coupled biochemical network of Nrf2, EMT and Notch. Binding between Notch ligands and receptors of neighboring cells give rise to cell-cell communication. (B) Snapshot of the multicell pattern after 120 hours of simulation starting from a randomized initial condition for a case of weak Nrf2 activation ( $g_{N R F 2}=0$ molecules/hour). Green, yellow and red hexagons depict epithelial (E), hybrid E/M and mesenchymal (M) cells, respectively. (C) Fraction of E, E/M, and $M$ cells as a function of distance from the 
bioRxiv preprint doi: https://doi.org/10.1101/2021.04.21.440858; this version posted April 22, 2021. The copyright holder for this preprint (which was not certified by peer review) is the author/funder. All rights reserved. No reuse allowed without permission.

invading edge. (D-E) Same as (B-C) for intermediate Nrf2 activation ( $g_{N R F 2}=0.510^{5}$ molecules/hour). (F-G) Same as (B-C) for strong Nrf2 activation ( $g_{N R F 2}=10^{5}$ molecules/hour). 
A
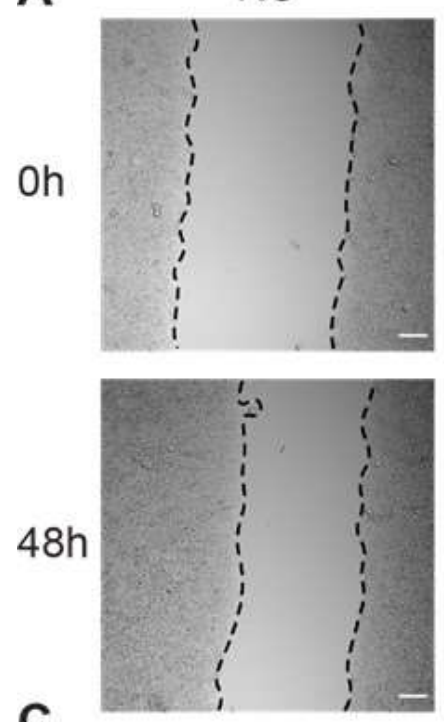

C

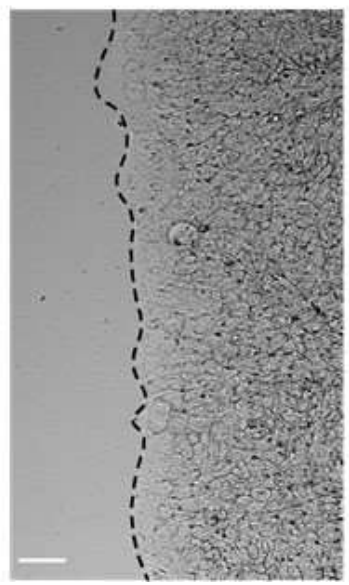

WT
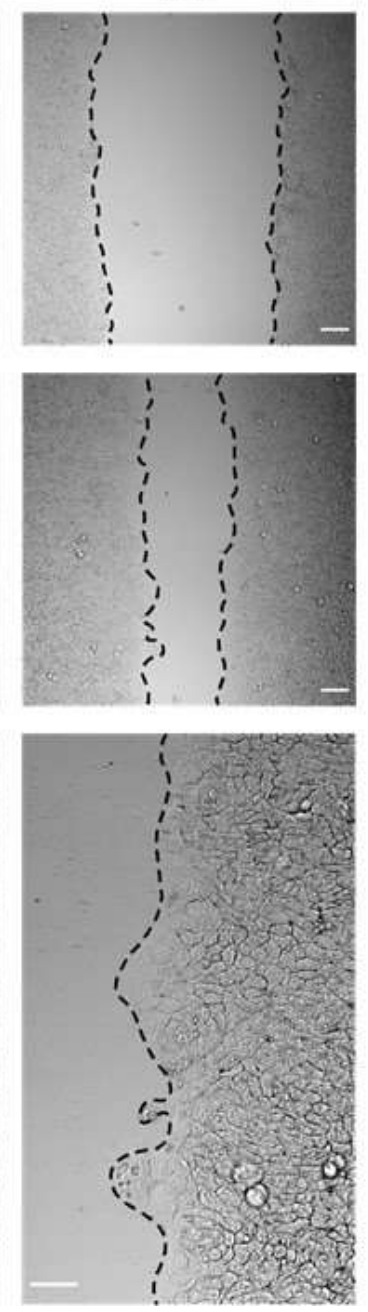

SFN
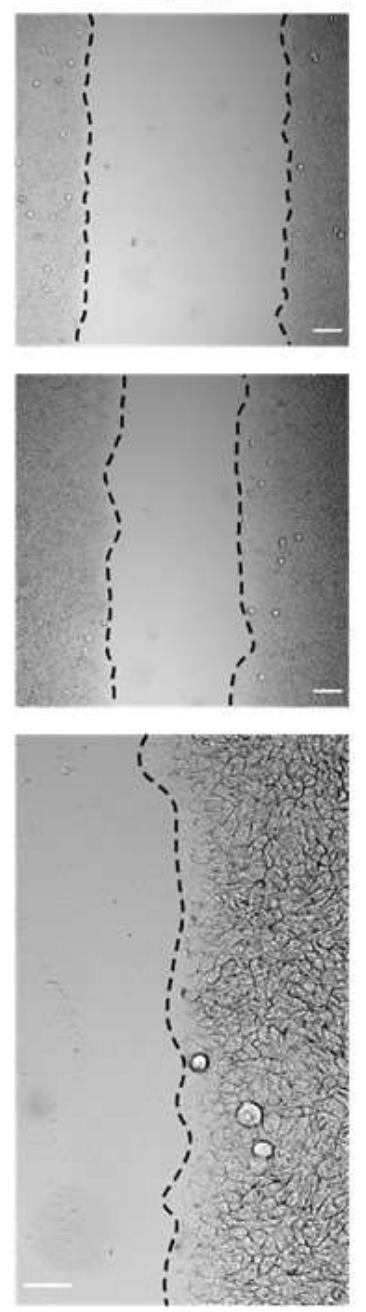

B

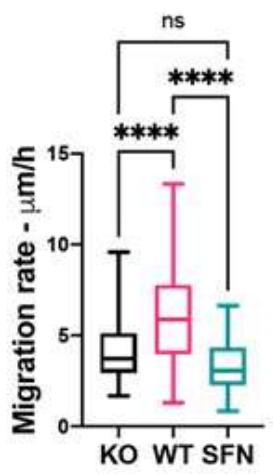

D

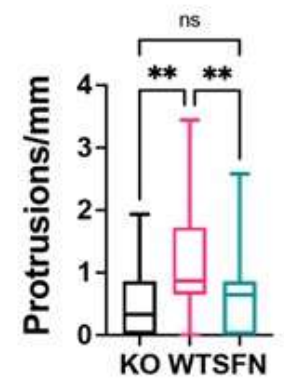

Figure 3. Nrf2 impairs collective migration in a 2D wound healing model. (A) Bright-field images of CRISPR/Cas9 NFE2L2-KO Pool RT4 cells (KO), wild-type RT4 cells (WT) and sulforaphane treated RT4 cells (SFN) for $0 \mathrm{~h}$ and $48 \mathrm{~h}$ migration time points. Scale bars, $100 \mu \mathrm{m}$. (B) Boxplot of migration rate for KO, WT, and SFN, respectively. (C) Representative images illustrating the formation of migration tips. Scale bars, $100 \mu \mathrm{m}$. (D) Boxplot of protrusion tips per milimeter for KO, WT, and SFN, respectively. The nonparametric Kruskal-Wallis test along with the Dunn's multiple comparisons test were used to compare across groups and the ROUT method was used to identify outliers in Figure 3D. For each condition $n>40$ scratch wound experiments. ns: not significance, $* *$ p-value $<0.01$, and $* * * *$-value $<0.0001$. 

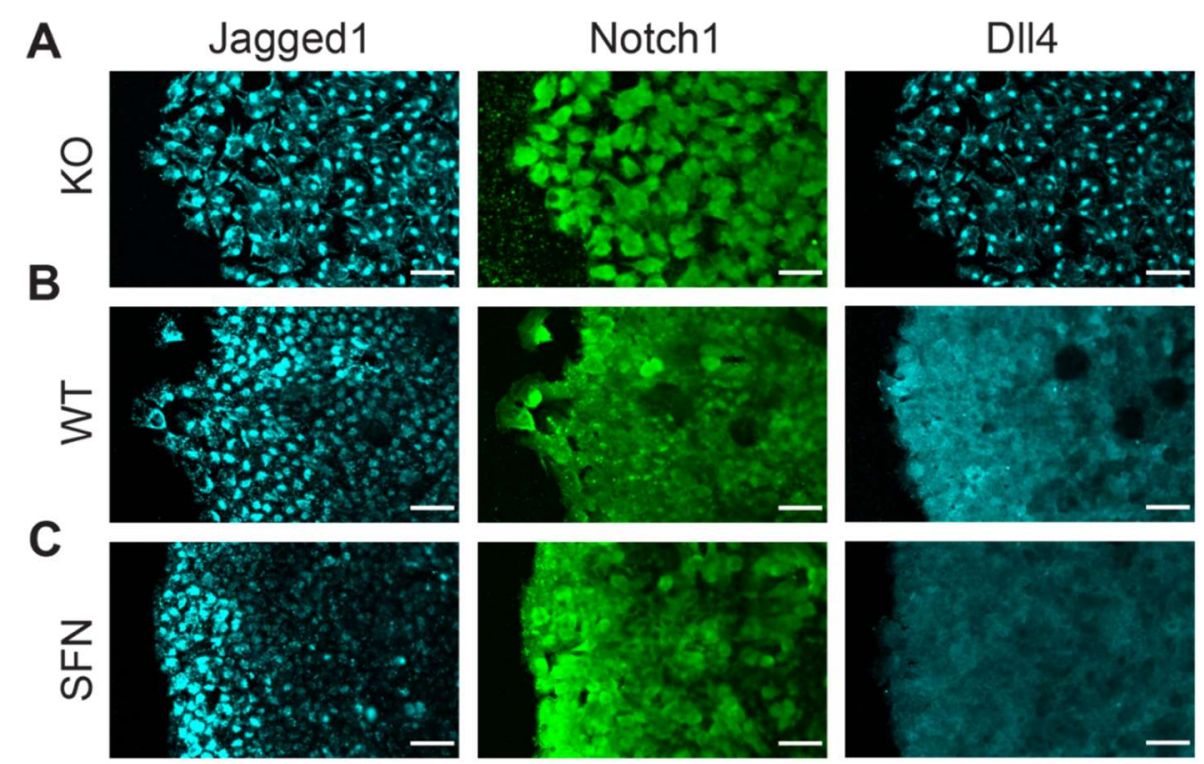

D
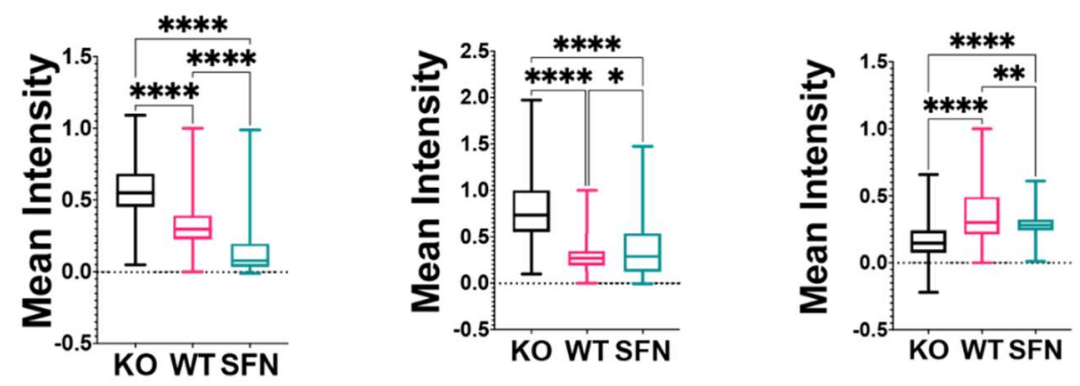

$\mathbf{E}$

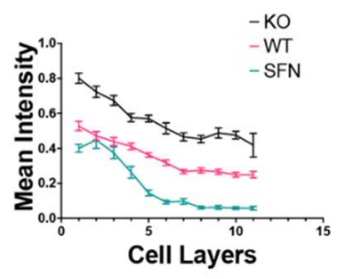

$\mathbf{F}$
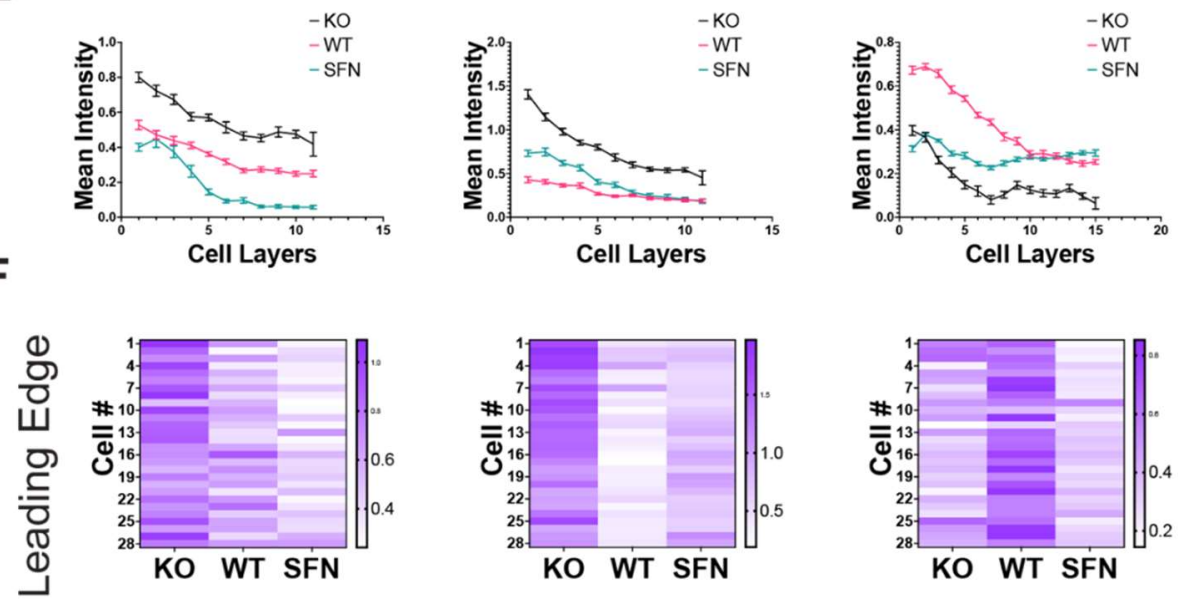

Figure 4. Nrf2 regulates the Notch signaling pathway near the wound edge. (A-C) Immunocytochemistry of

RT4 bladder cancer cells measuring (A) Jagged1, (B) Notch1, and (C) DII4 protein levels in a wound healing assay for CRISPR/Cas9 NFE2L2-KO Pool RT4 cells (KO), wild-type RT4 cells (WT) and sulforaphane treated RT4 cells (SFN), respectively. Scale bars, $50 \mu \mathrm{m}$. (D-F) Quantification of immunocytochemistry data. (D) Average intensity over the whole cell layer, (E) tracing of relative fluorescence intensity per tissue depth measured as 
number of cell layers, and (F) heatmap of representative cells near the wound leading edge for Jagged1, Notch1, and DII4, respectively. The nonparametric Kruskal-Wallis test along with the Dunn's multiple comparisons test were used to compare across groups (view Materials and Methods section). For each experiment $n>500$ cells per condition. ns: not significance, ${ }^{*} p$-value $<0.05, * * p$-value $<0.01$, and $* * * * p$ value $<0.0001$ 

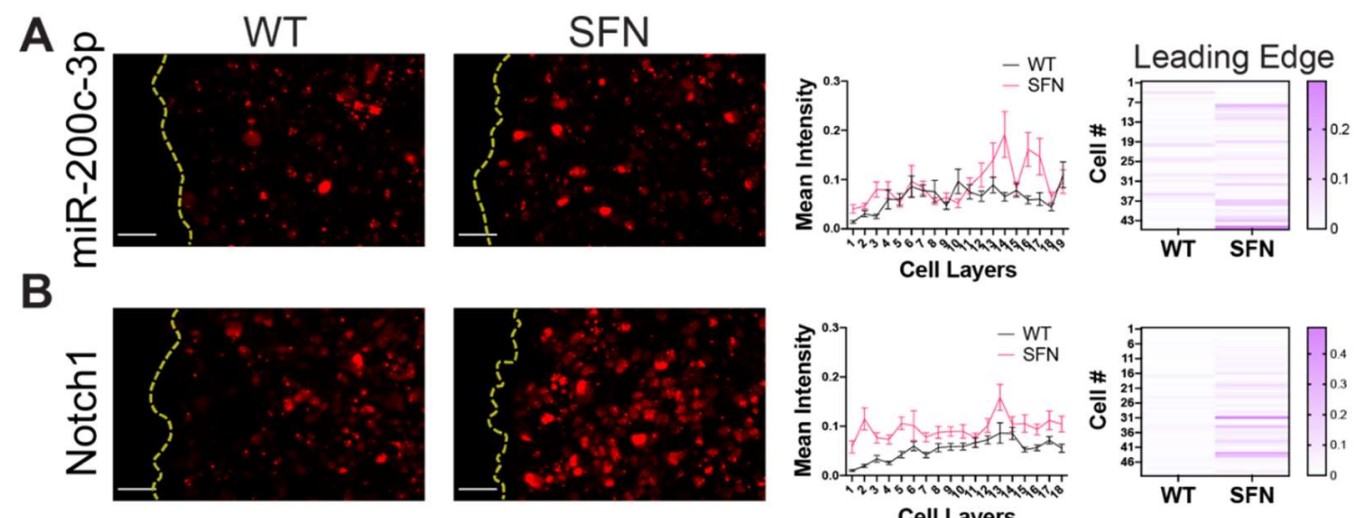

C
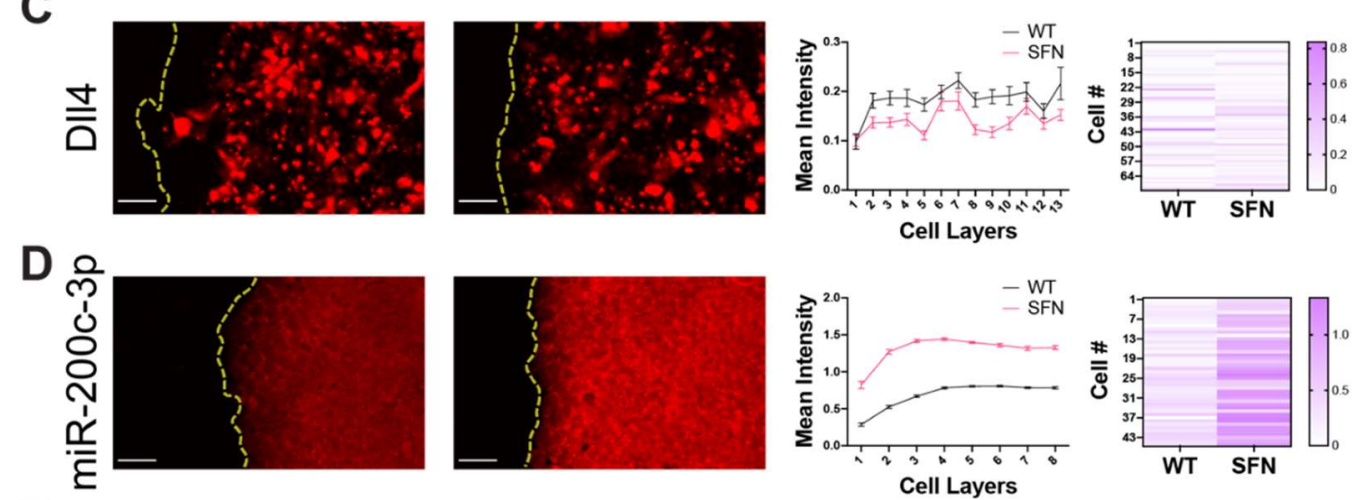

E
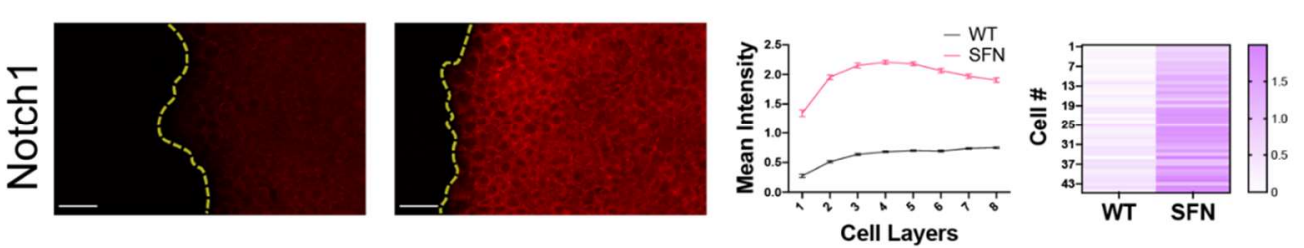

$\mathbf{F}$
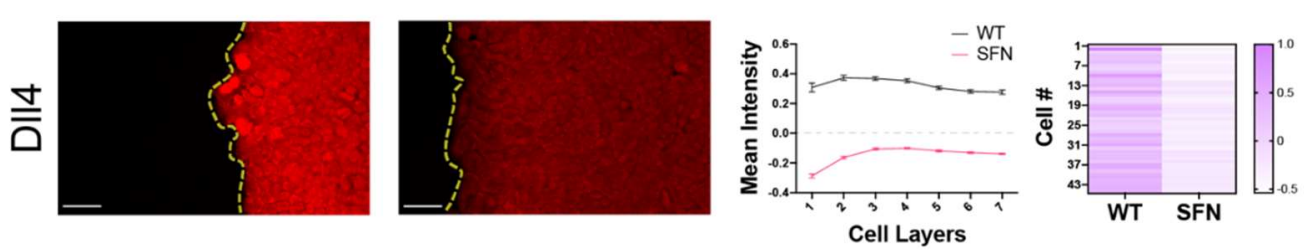

Figure 5. Nrf2 upregulation modulates microRNA miR-200c-3p and Notch1 and DIl4 mRNA near the wound edge. (A-C) Live single-cell gene expression measurements with the dsLNA probes in RT4 cells. From left to right: fluorescence images of WT and SFN cases, tracing of relative fluorescence intensity per tissue depth measured as number of cell layers, and heatmap of representative cells near the wound leading edge measuring microRNA miR-200c-3p (A), Notch1 mRNA (B), and DIl4 mRNA (C) levels in a scratch wound healing assay. (D-F) FISH assay in fixed RT4 cells. From left to right: fluorescence images of WT and SFN cases, tracing of relative fluorescence intensity per tissue depth measured as number of cell layers, and heatmap of 
representative cells near the wound leading edge measuring microRNA miR-200c-3p (D), Notch1 mRNA (E), and DII4 mRNA (F) levels in a scratch wound healing assay. Scale bars, $50 \mu \mathrm{m}$. The 2-way ANOVA with the post-hoc Tukey test to compare across groups was performed as well as the nonparametric Mann-Whitney test. For each experiment $n>500$ cells per condition. 


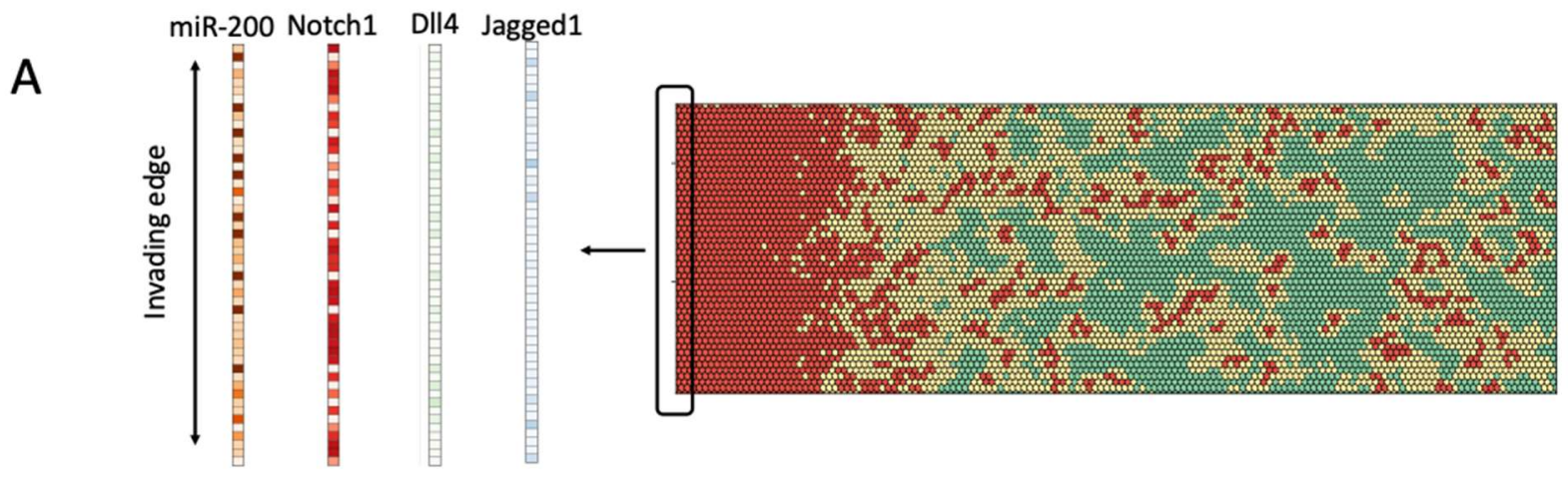

B

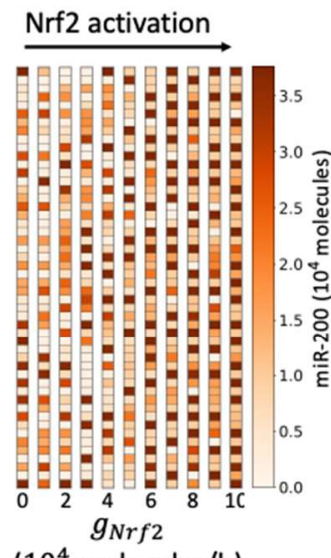

$\left(10^{4}\right.$ molecules/h)

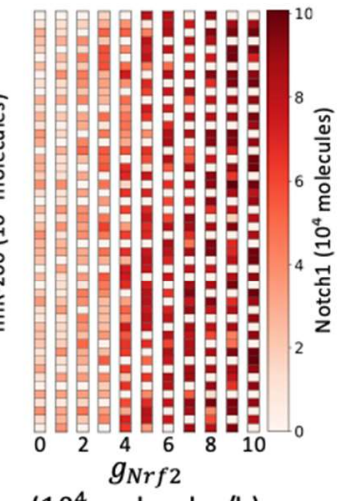

$\left(10^{4}\right.$ molecules/h)

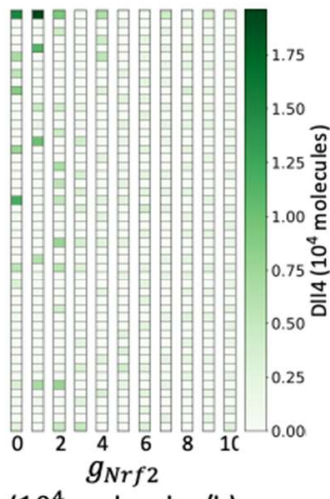

$\left(10^{4}\right.$ molecules/h)

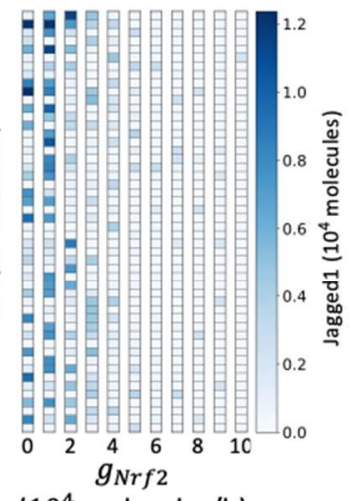

$\left(10^{4}\right.$ molecules/h)
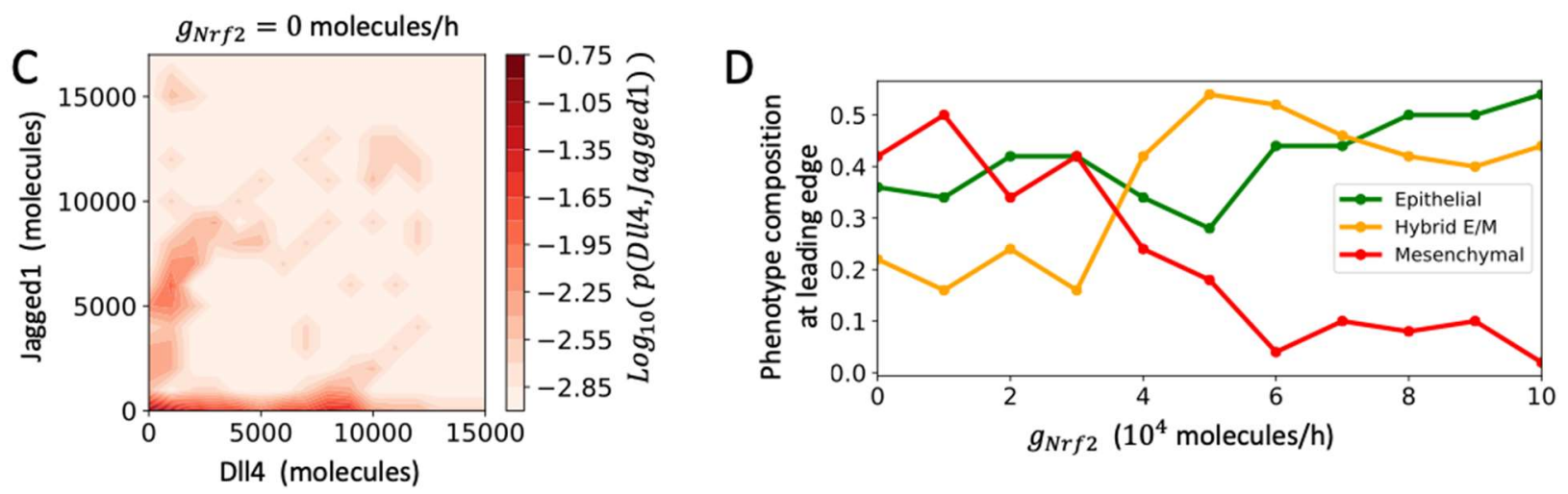

Figure 6. Analysis of the invading edge by the multicell model. (A) To conduct invading layer analysis, the expression of miR-200, Notch, Dll4 and Jagged1 is analysed in the leftmost layer of cells (i.e., the cell layer more exposed to EMT-inducing signal). (B) Heatmap of expression levels for miR-200, Notch1, DII4 and Jagged1 in the invading layer as a function of Nrf2 activation. (C) Log-normalized probability to observe cells with varying levels of DII4 and Jag4 in the invasive edge under low Nrf2 induction. (D) Fraction of Epithelial, 
bioRxiv preprint doi: https://doi.org/10.1101/2021.04.21.440858; this version posted April 22, 2021. The copyright holder for this preprint (which was not certified by peer review) is the author/funder. All rights reserved. No reuse allowed without permission.

hybrid E/M, and Mesenchymal cells in the invading layer as a function of Nrf2 activation. For panels C-D, results are averaged over 10 simulations starting from randomized initial conditions. 
A
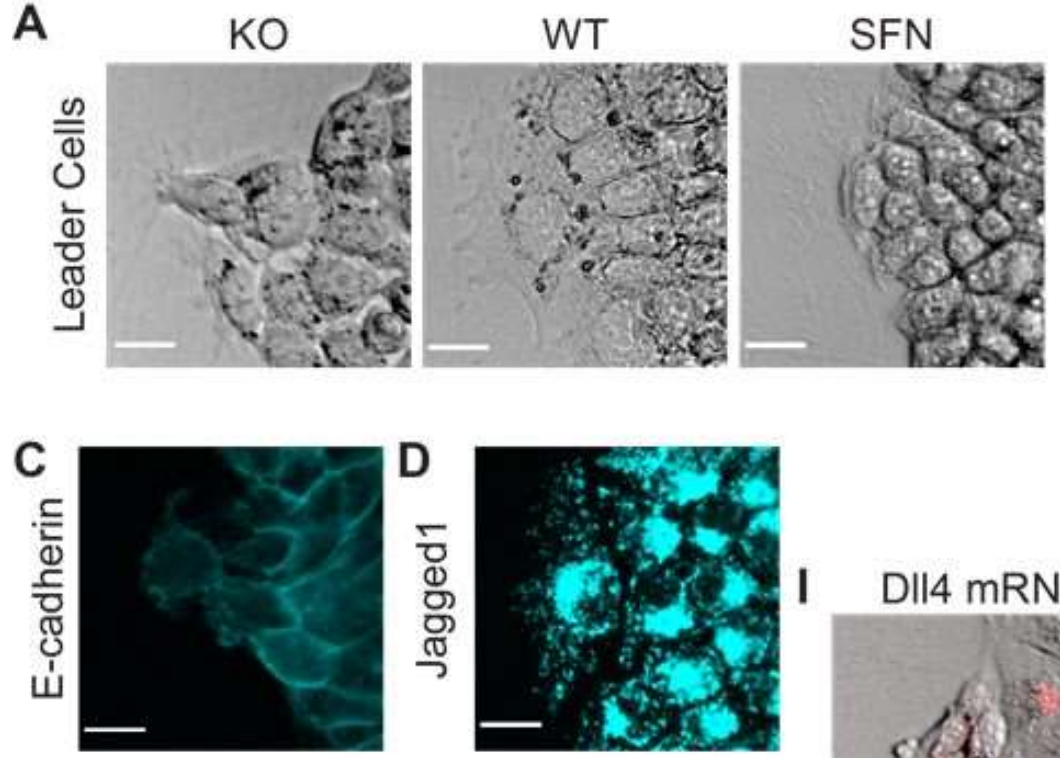

E
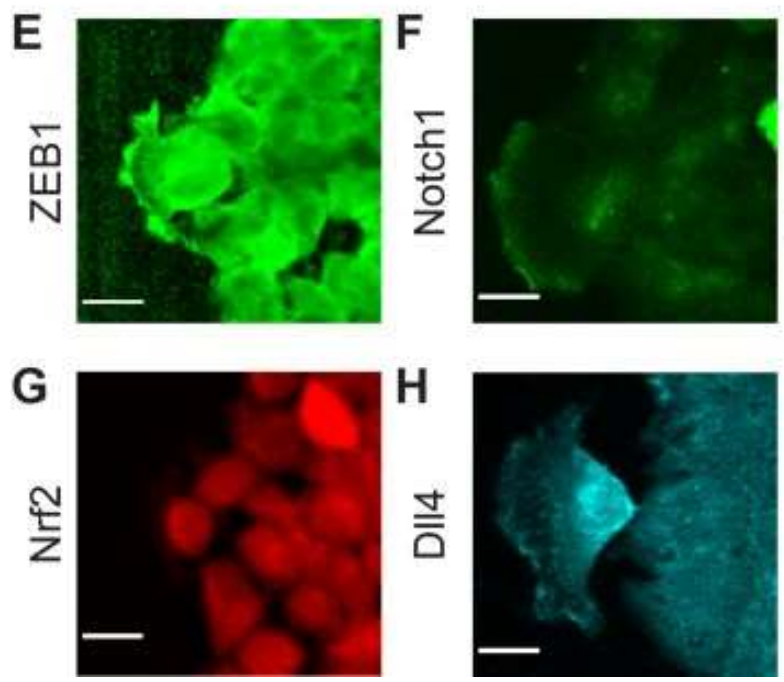

B

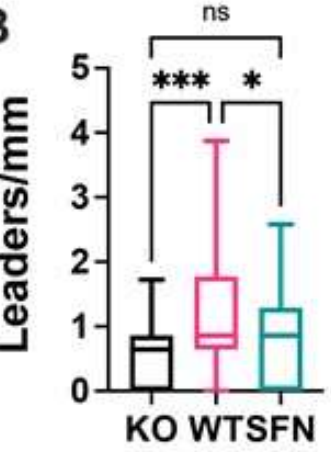

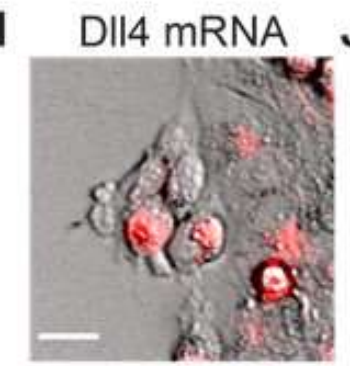

J Notch1 mRNA

K

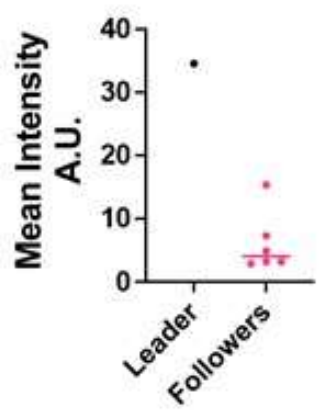

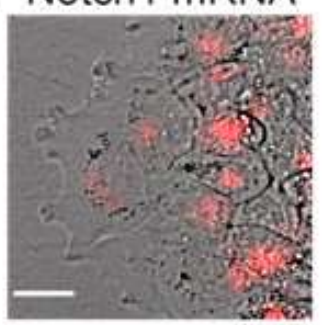

$\mathbf{L}$

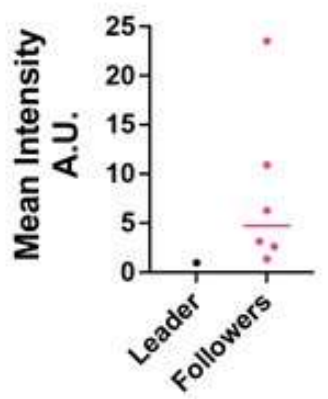

Figure 7. Leader cell formation near the leading edge. (A) Representative bright-field images of leader cells after a 24h wound healing assay for CRISPR/Cas9 NFE2L2-KO Pool RT4 cells (KO), wild-type RT4 cells (WT) and sulforaphane treated RT4 cells (SFN), respectively. (B) Bar chart showing leader cells per millimeter for CRISPR/Cas9 NFE2L2-KO Pool RT4 cells (KO), wild-type RT4 cells (WT) and sulforaphane treated RT4 cells (SFN), respectively. ns: not significance, $*$ p-value $<0.05$, and $* * *$ p-value $<0.001$. (C-H) Representative immunocytochemistry images of WT leader cells characterizing gene expression for (C) E-cadherin, (D) Jagged1, (E) ZEB1, (F) Notch1, (G) Nrf2, (H) Dll4. (I-J) Representative composite images using the dsLNA biosensors to measure mRNA levels of (I) DII4 and (J) Notch1, respectively. Mean intensity of leader vs 
bioRxiv preprint doi: https://doi.org/10.1101/2021.04.21.440858; this version posted April 22, 2021. The copyright holder for this preprint (which was not certified by peer review) is the author/funder. All rights reserved. No reuse allowed without permission.

follower cells for levels of (K) DII4 mRNA and (L) Notch1 mRNA. Scale bars, $20 \mu \mathrm{m}$. The nonparametric KruskalWallis test along with the Dunn's multiple comparisons test were used to compare across groups and the ROUT method was used to identify outliers in Figure 7B. For each condition $n>40$ scratch wound experiments. 